\title{
Study of Two Dimensional Electron Gas Formation at AlGaN/GaN Interfaces using Kelvin Probe Force Microscopy
}

\author{
P. Caban 1 , R. Thorpe ${ }^{2}$, L. Feldman ${ }^{2}$, K. Pedersen ${ }^{3}$ and V.N. Popok, ${ }^{3, *}$
}

1) Institute of Electronic Materials Technology, 01-919 Warsaw, Poland
2) Department of Physics \& Astronomy, Rutgers The State University of New Jersey, 08854 Piscataway, USA
${ }^{3)}$ Department of Materials and Production, Aalborg University, 9220 Aalborg, Denmark
${ }^{*}$ E-mail vp@mp.aau.dk

$\mathrm{AlGaN} / \mathrm{GaN}$ heterostructures are of high research and industrial interest for the production of high electron mobility transistors utilizing the two-dimensional electron gas (2DEG) induced at the interface due to polarization effects. Critical AlGaN thickness is an important practical parameter which is under the study in this work.

Ultrathin layers of AlGaN (between 2-12 nm thick) are grown on top of $\mathrm{GaN}$ in order to study 2DEG formation. The structures are prepared using the metal organic chemical vapour deposition method. Combining Rutherford backscattering, X-ray photoelectron spectroscopy and ion sputtering the composition and thickness of the AlGaN layers are found. The measured thicknesses agree well with the expected values. For selected samples, TEM analysis is also performed showing quality of the interfaces. Al/Ga ratio is found to be nearly constant, 0.26-0.28, in layers thicker than 5-6 nm. In the thinner films, estimation of the composition is unreliable due to the photoelectrons coming from the underlying GaN film. Kelvin probe force microscopy (KPFM) is used to study surface potential depending on AlGaN layer thickness.
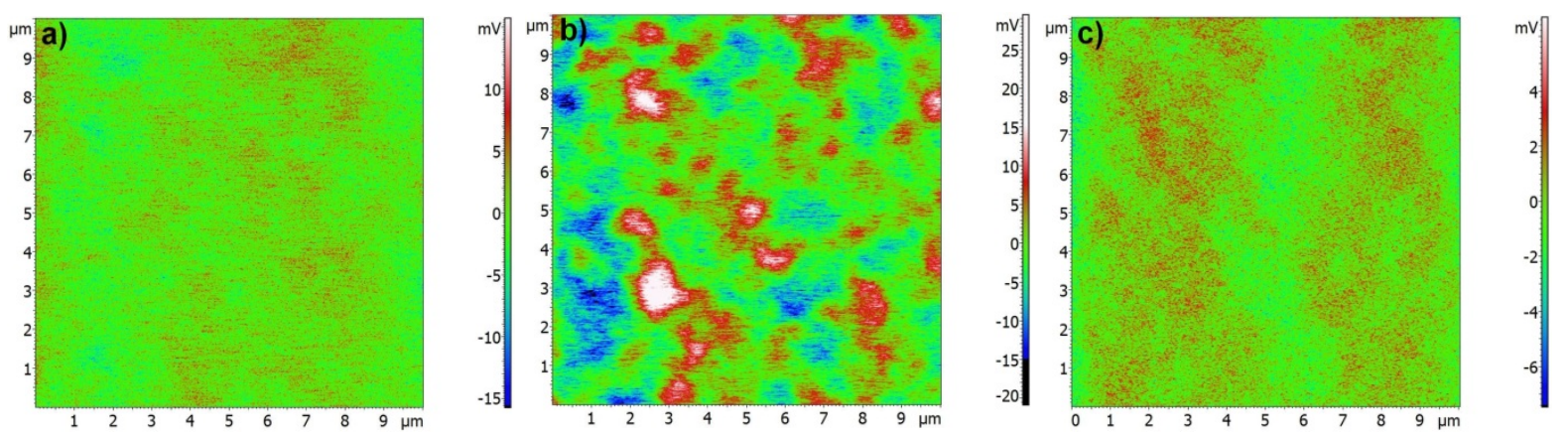

Fig. 1. KPFM images of (a) GaN, (b) $\mathrm{AlGaN}(3.5 \mathrm{~nm}) / \mathrm{GaN}$ and (c) $\mathrm{AlGaN}(12 \mathrm{~nm}) / \mathrm{GaN}$

Pure GaN samples show homogeneous potential distribution across the surface (see Fig. 1a) with a mean value of $0.72 \pm 0.3 \mathrm{~V}$. This corresponds to an upward band bending of $1.1 \pm 0.1 \mathrm{~V}$. Both values are in good agreement with previous measurements [1]. Overall, the contact potential difference rises from pure $\mathrm{GaN}$ to $\mathrm{AlGaN} / \mathrm{GaN}$ following the theoretical estimates for these compound semiconductors. Growth of thin (ca. 2 and $4 \mathrm{~nm}$ ) layers of AlGaN leads to a mosaic-like potential distribution indicating that 2DEG is formed at the interface but it is not continuous because of the low layer thickness and poor stoichiometry (see Fig. 1b). Homogeneity of the surface potential maps increases for layers thicker than 7-8 nm (Fig. 1c) allowing to conclude about critical thickness of $\mathrm{AlGaN}$ for the formation of continuous 
2DEG. Similar findings on critical thickness of a barrier layer for the formation of 2DEG were reported using KPFM for $\mathrm{LaAlO}_{3} / \mathrm{SrTiO}_{3}$ heteroiterfaces [2]. The obtained results are also in good correlation with earlier data reporting 2DEG observations for AlGaN layers thicker than $3.5 \mathrm{~nm}$ in which the mobility and charge carrier concentration increase reaching maximal values for the layers at around $10 \mathrm{~nm}[3,4]$. Thus, the work brings important insights into registration of $2 \mathrm{DEG}$ at $\mathrm{AlGaN} / \mathrm{GaN}$ interfaces using KPFM.

\section{References.}

[1] S. Chevtchenko, X. Ni, Q. Fan, A.A. Baski, H. Morkoc, Appl. Phys. Lett. 88, 122104 (2006).

[2] V.N. Popok, A. Kalabukhov, R. Gunnarsson, S. Lemeshko, T. Claeson, D. Winkler, J. Adv. Microsc. Res. 5, 26-30 (2010).

[3] J.P. Ibbetson, P.T. Fini, K.D. Ness, S.P. DenBaars, J.S. Speck, U.K. Mishra, Appl. Phys. Lett. 77, 250-252 (2000).

[4] S. Heikman, S. Keller, Y. Wu, J.S. Speck, S.P. DenBaars, U.K. Mishra, J. Appl. Phys. 93, 10114-10118 (2006). 